\title{
Immobilization of polyethylene oxide surfactants for non-fouling biomaterial surfaces using an argon glow discharge treatment
}

\author{
M.-S. Sheu , A.S. Hoffman , B.D. Ratner , J. Feijen \& J.M. Harris
}

To cite this article: M.-S. Sheu , A.S. Hoffman , B.D. Ratner , J. Feijen \& J.M. Harris (1993) Immobilization of polyethylene oxide surfactants for non-fouling biomaterial surfaces using an argon glow discharge treatment, Journal of Adhesion Science and Technology, 7:10, 1065-1076, DOI: 10.1163/156856193X00583

To link to this article: https://doi.org/10.1163/156856193X00583

\section{Published online: 02 Apr 2012.}

\section{Submit your article to this journal $\sqsubset$}

Џ Article views: 26

Citing articles: 42 View citing articles $匚$ 


\title{
Immobilization of polyethylene oxide surfactants for non-fouling biomaterial surfaces using an argon glow discharge treatment
}

\author{
M.-S. SHEU, ${ }^{1, *}$ A. S. HOFFMAN, ${ }^{1}+$ B. D. RATNER, ${ }^{1}$ J. FEIJEN ${ }^{2}$ and \\ J. M. HARRIS ${ }^{3}$ \\ ${ }^{1}$ Center for Bioengineering, FL-20, University of Washington, Seattle, WA 98195, USA \\ ${ }^{2}$ Department of Chemical Engineering, University of Twente, P.O. Box 217, 7500 AE, Enschede, \\ The Netherlands \\ ${ }^{3}$ Department of Chemistry, University of Alabama, Huntsville, AL 35899, USA
}

Revised version received 26 April 1993

\begin{abstract}
A non-fouling (protein-resistant) polymer surface is achieved by the covalent immobilization of polyethylene oxide (PEO) surfactants using an inert gas discharge treatment. Treated surfaces have been characterized using electron spectroscopy for chemical analysis (ESCA), static secondary ion mass spectrometry (SSIMS), water contact angle measurement, fibrinogen adsorption, and platelet adhesion. This paper is intended to review our recent work in using this simple surface modification process to obtain wettable polymer surfaces in general, and non-fouling biomaterial surfaces in particular.
\end{abstract}

Keywords: Surface modification; glow discharge treatment; non-fouling surfaces; wettable polymer surfaces; poly(ethylene oxide) surfactants.

\section{INTRODUCTION}

Surfaces modified with polyethylene oxide (PEO) exhibit resistance to fouling by protein adsorption and platelet adhesion [1,2], mainly due to the non-ionic hydrophilic characteristics (high water content) and the large excluded volume of the PEO molecule [3] as well as its high chain mobility in water [4]. Such non-fouling surfaces are important to most biotechnological and medical applications, such as diagnostic assays, drug-delivery systems, biosensors, bioseparations, and implants and medical devices. As a result, a wide variety of surface treatments for generating $\mathrm{PEO}$-containing surfaces have been investigated, such as physical adsorption [5-8], surface entrapment $[9,10]$, chemical immobilization [11, 12], surface grafting [4, 13], and plasma (or glow discharge) polymerization [14], shown schematically in Fig. 1 [15]. Bulk polymers containing PEO have also been prepared as block copolymers [16-19] and selfcrosslinked hydrogels [20] (see also Fig. 1). Many of these surface modification methods have limitations, such as physical or chemical instability, lack of functional groups, low surface coverage, undesirable changes of the bulk properties of the substrate, multiple or costly process steps, or extreme reaction conditions.

*Present address: Advanced Surface Technology, Inc., 9 Linnell Circle. Billerica, MA 01821, USA.

†To whom correspondence should be addressed. 


\section{Surface Modifications to Obtain PEO Surfaces}

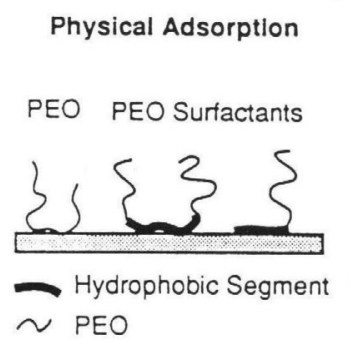

Surface Entrapment

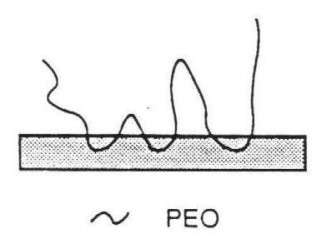

Chemical Immobillzation

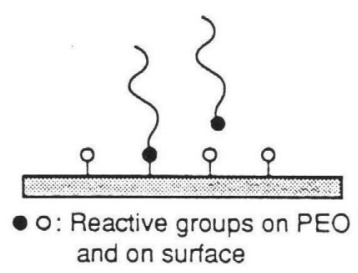

Plasma Deposition

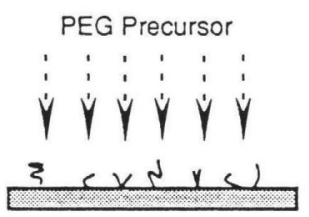

Surface Grafting

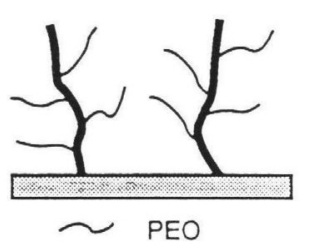

Surface Crosslinking by Plasma Treatment

PEO Surfactants

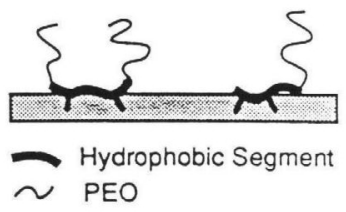

Bulk Modifications to Obtain PEO Surfaces

Block Copolymerization

Self-Crosslinking
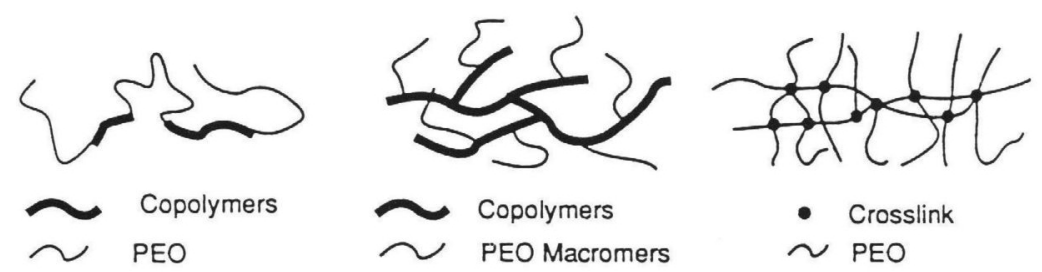

Figure 1. Schematic diagram of the surface and bulk modifications of polymers used to yield PEO surfaces [15].

Glow discharge processes have been widely used for the surface modification of polymers, mainly due to their localized surface treatment without changing the bulk properties of the polymer. Recently, the plasma polymerization (deposition) process has shown great potential to modify the surface composition of polymers by forming a deposited polymer layer or an organic thin film. In this process, the polymerizable gases are used and can be introduced to the plasma reactor during or after the glow discharge treatment. In principle, the surface chemistry of the modified substrate can be tailored by selecting the proper monomers or organic precursors in the glow discharge process. However, due to the complexity of the plasma reactions, it is difficult to obtain a specific or desired surface chemistry in 
the plasma-deposited layers. Also, the treatment is usually limited to using small volatile molecules in the plasma.

A modification of the plasma polymerization process was developed to graft/ polymerize non-volatile monomers using plasma treatment [21, 22]. In this process, monomers, mainly acrylates, were first adsorbed or coated on the substrate from solutions. An inert gas plasma treatment was then applied to initiate the grafting polymerization on the precoated substrate. The rate of polymerization or grafting was also enhanced by adding conventional freeradical initiators [23], suggesting a free-radical mechanism for the graft polymerization. However, if the monomers used are incompatible with the substrate and form an uneven precoated laycr before plasma treatment, then an uneven surface coating on the treated substrate will result.

In the mid-1960s, Schonhorn and co-workers improved the adhesion strength of polymers using a plasma treatment with inert gases [24, 25]. Their treatment was based on the premise that the adhesion strength of polymers is mainly limited by the presence of 'weak boundary layers' on surfaces, e.g. lowmolecular-weight polymers and impurities. When treated with inert gas plasmas, these weak boundary layers can be crosslinked to the larger molecules in the surface, and this enhances the adhesion strength of the treated surface. This process was called the 'CASING' technique (Crosslinking by Activated Species of INert Gases) [24, 25]. Such a plasma treatment process is a simple and convenient way to effect covalent crosslinking within a thin layer at a substrate surface.

In our laboratory, we have developed a process which extends the CASING technique to the covalent immobilization of surface-active compounds on hydrophobic polymer surfaces. This is shown schematically in Fig. 2 [15]. In this process, surface-active compounds (e.g. PEO surfactants) are first absorbed or deposited from aqueous or organic solutions onto polymer films. These precoated surfactants, acting as the 'weak boundary layer', are then crosslinked to the surfaces and to each other by an inert gas discharge treatment (e.g. argon). This glow discharge immobilization process shows unique advantages: a fast and simple two-step process; covalent immobilization; no change in the bulk properties of the polymer substrate; low dependency on the surface composition of the polymer substrate; high and uniform surface coverage; and no need or requirement to use volatile organic vapors.

A non-fouling polymer surface was prepared using an inert gas discharge treatment of a low-density polyethylene (LDPE) surface which had been precoated with an oleyl PEO surfactant (Brij99) and polyethylene oxidepolypropylene oxide-polyethylene oxide (PEO-PPO-PEO) tri-block copolymer surfactants (Pluronic) [15, 26]. Electron spectroscopy for chemical analysis (ESCA) was used to estimate the retention of the PEO surfactants on the treated surfaces. The enhanced wettability of the modified surfaces was characterized using water contact angle measurements. The non-fouling properties of the treated surfaces were examined by adsorption of ${ }^{125}$ I-labeled baboon fibrinogen and in vitro adhesion of ${ }^{111}$ In-labeled baboon platelets. Static secondary ion mass spectrometry (SSIMS) was used as a complementary method to ESCA and water contact angle goniometry to characterize the glow-discharge-treated surfaces, particularly to correlate the surface structure of the treated surfactants, 


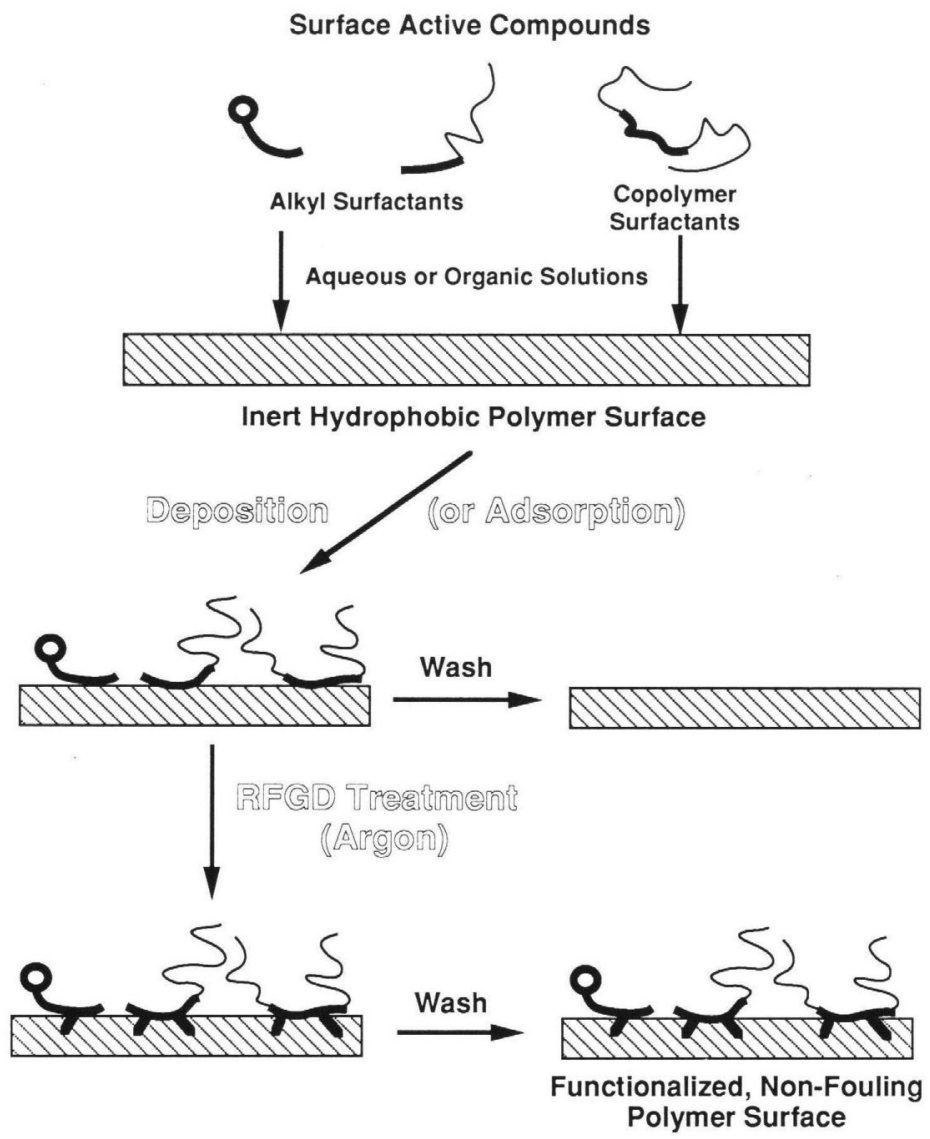

Figure 2. Schematic diagram of the argon glow discharge treatment used for the immobilization of surface-active compounds [15].

especially PEO chains, to the protein/platelet adsorption results. This paper is a review of our recent work in immobilizing $\mathrm{PEO}$ surfactants to generate a permanent non-fouling surface using an argon glow discharge treatment.

\section{EXPERIMENTAL}

\subsection{Materials}

Low-density polyethylene (LDPE) films (Cadillac Plastics, Seattle, WA) with an average thickness of about $0.3 \mathrm{~mm}$ were precleaned by sequential extraction with methylene chloride, acetone, and water for 15 min each in a sonicator. Brij99, an oleyl PEO (Sigma Chemical Co.), and Pluronic surfactants (gifts from BASF Corp.) were used as received. PEO (Sigma Chemical Co.) and PPO (Scientific Polymer Products, Inc.) homopolymers were selected as controls for comparing with the Brij99 and Pluronic surfactants. The chemical properties of the PEO, PPO, and PEO surfactants used are listed in Table 1.

Fibrinogen and platelets were purified from fresh baboon blood (from the Regional Primate Research Center, Seattle, WA) [27] and radiolabelled with ${ }^{125} \mathrm{I}$ 
Table 1.

Properties of the PEO, PPO polymers, and PEO surfactants

\begin{tabular}{lccl}
\hline Sample code & $\begin{array}{l}\text { Average } \\
\text { molecular weight }\end{array}$ & $\begin{array}{c}\mathrm{EO} / \mathrm{PO} / \mathrm{EO}^{\prime} \\
\text { (repeat units) }\end{array}$ & $\begin{array}{l}\text { Theoretical O/C } \\
\text { atomic ratio }\end{array}$ \\
\hline Brij99 & 1100 & \multicolumn{1}{c}{} & 0.36 \\
PEO1K & 1000 & $23(\mathrm{EO})$ & 0.50 \\
PEO10K & 10000 & $227(\mathrm{EO})$ & 0.50 \\
PPO4K & 4000 & $0 / 69 / 0$ & 0.33 \\
Pluronic121 & 4400 & $6 / 67 / 6$ & 0.36 \\
Pluronic122 & 5000 & $13 / 67 / 13$ & 0.37 \\
Pluronic127 & 11500 & $98 / 67 / 98$ & 0.44 \\
\hline
\end{tabular}

"EO: ethylene oxide unit; PO: propylene oxide unit.

${ }^{b}$ An oleyl ether with average 20 repeat units in $\mathrm{PEO}$ segments.

and ${ }^{111}$ In, respectively, according to previously published protocols [28, 29]. Deionized and distilled water was used in the contact angle measurements.

\subsection{Glow discharge immobilization of PEO surfactants}

The glow discharge immobilization of PEO surfactants on LDPE is a two-step process: surfactant deposition on polymer substrates followed by surface treatment with argon plasmas, as shown schematically in Fig. 2. Detailed procedures for the surfactant deposition, the glow discharge treatments, and the washing protocol have been described in previous publications and are briefly presented here [15]. PEO surfactants were physically deposited onto LDPE using a simple dip-coating method. LDPE films were dipped in $1 \%(\mathrm{w} / \mathrm{v})$ chloroform solutions of the surfactants for $30 \mathrm{~s}$. After drying overnight, the films were then treated with an argon glow discharge.

A capacitive radio-frequency glow discharge (RFGD) at $13.56 \mathrm{MHz}$ (HF-300, ENI Power Systems Inc., Rochester, NY) was used to treat the LDPE or PEO surfactant/LDPE surfaces in a glass-cylinder reactor $(11.5 \mathrm{~cm}$ inside diameter $\times 80 \mathrm{~cm}$ long). After evacuating the chamber three times to a base pressure of 5-7 mTorr, a static argon (Air Products and Chemicals Inc., Allentown, PA; pre-pure grade: $>99.95 \%$ ) gas discharge without flow was generated at a reactor pressure of $25 \mathrm{mTorr}$, a low power of $\leqslant 5 \mathrm{~W}$, and ambient temperature. The RFGD treatment time was varied from 0 to $300 \mathrm{~s}$.

After the treatment, the surfaces were washed in chloroform twice for $30 \mathrm{~min}$ each and then soaked in fresh chloroform overnight. The samples were then dried in air and stored in a laminar flow hood before further surface characterization. This washing protocol was performed to completely remove all of the physically precoated PEO surfactants from the treated surface.

\subsection{Surface characterization}

The RFGD-treated surfaces were characterized by ESCA, SSIMS, and water contact angle measurements. ESCA measurements were done on an SSX-100 spectrometer (Surface Science Instruments, Mountain View, CA) using a monochromatic $\mathrm{Al} K_{a} \mathrm{X}$-ray source with a $5 \mathrm{eV}$ floodgun. The $\mathrm{X}$-ray spot size 
(analyzing area) on the sample surfaces was about $1000 \mu \mathrm{m}$ in diameter. A standard $55^{\circ}$ take-off angle (the angle between the surface normal and the axis of the analyzer lens) was used for all measurements. Surface oxygen to carbon ratios $(\mathrm{O} / \mathrm{C})$ from survey scans and the other carbon peak $(286.4 \mathrm{eV})$ in highresolution $\mathrm{C} 1 \mathrm{~s}$ spectra were used to detect the presence of PEO surfactants on the treated surfaces. A Ramé-Hart goniometer $(\mathrm{A}-100)$ was used to measure the advancing water contact angles on the treated films at room temperature in air.

SSIMS analysis was performed on the SSX-100 surface analysis system equipped with a static SIMS add-on (SubMonolayer System, Mountain View, CA). The primary ion source was a $3.5 \mathrm{keV}, 1.5 \mathrm{nA} \mathrm{Xe}{ }^{+}$beam. Positive-ion SSIMS spectra for the treated films were recorded from $\mathrm{m} / z=0$ to 100 and three samples for each surface were measured. In order to observe the relative amount of PEO to hydrocarbon on the treated surface, a PEO index is defined as the ratio of intensities from the sum of two SSIMS peaks, each pair of peaks being characteristic of either the PEO $(m / z=45$ and 89$)$ or the LDPE $(m / z=41$ and 55) $[30]$ :

$$
\mathrm{PEO} \text { index }=\frac{m / z(45)+m / z(89)}{m / z(41)+m / z(55)}
$$

\subsection{Fibrinogen adsorption and in vitro platelet adhesion}

Polymer films $(15 \mathrm{~mm} \times 11 \mathrm{~mm})$ were prehydrated citrated phosphate-buffered saline with $0.02 \%$ sodium azide and $0.01 \mathrm{M}$ sodium iodide (CPBSzi) at $37^{\circ} \mathrm{C}$ for $4 \mathrm{~h}$. Then an ${ }^{125} \mathrm{I}$-labeled baboon fibrinogen solution was added to reach a $0.2 \mathrm{mg} / \mathrm{ml}$ total protein concentration (average counts $=2 \times 10^{\dagger} \mathrm{cpm} / \mathrm{mg}$ fibrinogen) and the films were incubated at $37^{\circ} \mathrm{C}$ for $2 \mathrm{~h}$. After incubation, the proteinadsorbed films were washed with $100 \mathrm{ml}$ of fresh CPBSzi and then counted in a gamma counter. The amount of protein adsorbed per unit area was calculated from the specific activity of the fibrinogen and the planar surface area of films.

In the in vitro platelet-adhesion experiments, after prehydration in CPBSzi, the samples were immersed in $1 \times 10^{8}{ }^{111}$ In-labeled platelets per $\mathrm{ml}$ at $37^{\circ} \mathrm{C}$ for $2 \mathrm{~h}$. Detailed protocols for protein adsorption and platelet adhesion have been described previously [15,29].

\section{RESULTS AND DISCUSSION}

Figures 3 and 4 show the results of ESCA and advancing water contact angle measurements on the RFGD-treated/ $\mathrm{CHCl}_{3}$-washed surfaces, respectively [26]. Without RFGD treatment, i.e. RFGD treatment time at $0 \mathrm{~s}$, the physically deposited surfactants are completely removed from the $\mathrm{CHCl}_{3}$-washed surfaces; these washed surfaces are similar to the untreated LDPE control. With RFGD treatment, on the other hand, significant increases in the surface $\mathrm{O} / \mathrm{C}$ and the surface wettability (water contact angle $<30^{\circ}$ ) at all treatment times $(15-300 \mathrm{~s})$ were found. These reveal that the PEO surfactants are retained on the RFGDtreated surfaces. Even after extensive washing in chloroform for 4 days, the surfaces remained the same, i.e. high $\mathrm{O} / \mathrm{C}$ ratios $(0.29)$ and low water contact angles $\left(<30^{\circ}\right)$. This strong retention of the surfactants on the treated surfaces 


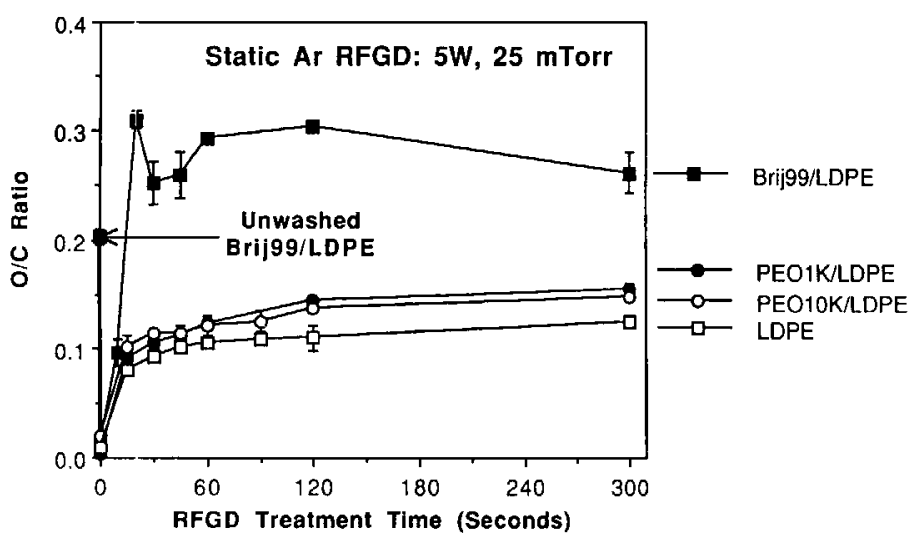

Figure 3. ESCA O/C atomic ratios for LDPE, PEO/LDPE, and Brij99/LDPE surfaces after Ar RFGD treatment and washing in chloroform [26]. Number of samples, $n$, equals 3 .

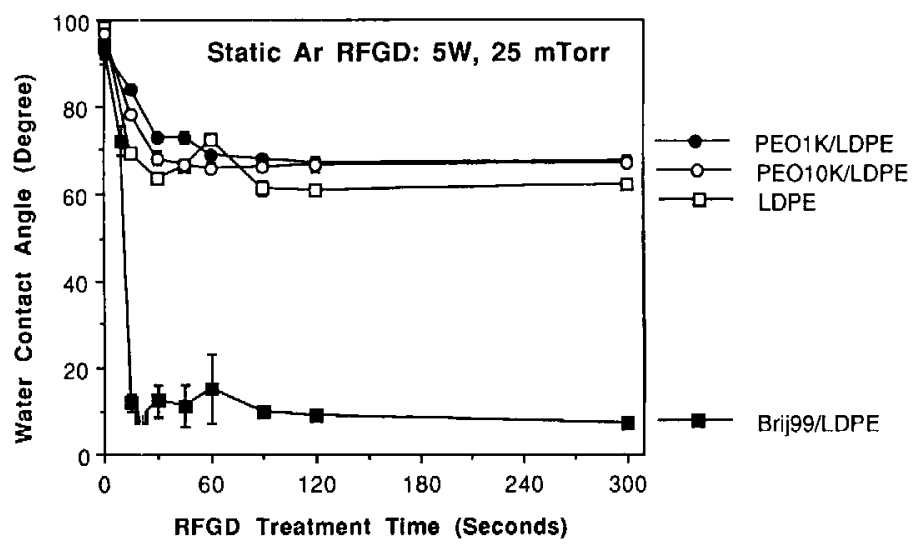

Figure 4. Advancing water contact angles on LDPE, PEO/LDPE, and Brij99/LDPE surfaces after Ar RFGD treatment and washing in chloroform [26]. Number of samples, $n$, equals 5 .

suggests that the immobilization of PEO surfactants is probably not due to physical adsorption and/or surface entrapment.

In addition, the ether carbons in the high-resolution $\mathrm{C} 1 s$ spectra on the RFGD-treated/ $\mathrm{CHCl}_{3}$-washed Pluronic/LDPE surfaces increase with increasing chain length of PEO in the surfactants, as shown in Fig. 5 [15]. This is another indication of the presence of the surfactants on the treated/washed surfaces. In contrast, only minor increases in surface oxidation and wettability were observed on the RFGD-treated $\mathrm{CHCl}_{3}$-washed $\mathrm{LDPE}$ and PEO/LDPE control surfaces. The latter clearly demonstrates that PEO homopolymers cannot be immobilized by this method. This inefficient immobilization of PEO homopolymers suggests that the glow discharge immobilization of the PEO surfactants on the LDPE films may be through the hydrophobic segments rather than the PEO segments.

Both static (without argon gas flow) and dynamic (with argon gas flow) RFGD have also been compared under the same treatment power and reactor pressure. 


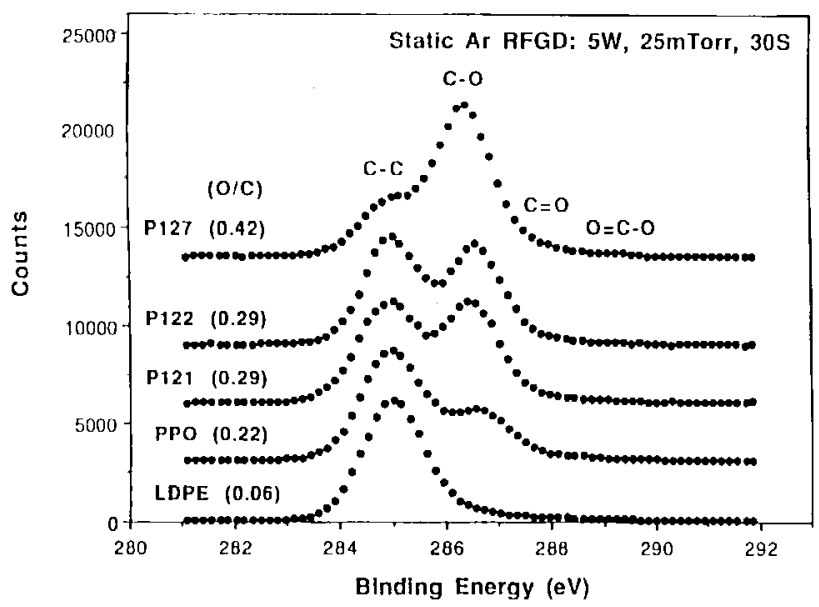

Figure 5. ESCA high-resolution $\mathrm{C} 1 s$ spectra for LDPE, PPO/I.DPE, and Pluronic/LDPE surfaces after Ar RFGD treatment for $30 \mathrm{~s}$ and washing in chloroform [15]. P denotes Pluronic.

However, no significant differences in the surface $\mathrm{O} / \mathrm{C}$ ratios were found for either the LDPE or the Brij99/LDPE surfaces. This lack of influence of gas flow in the surfactant immobilization is due to the inert properties of the argon gas, which is not consumed by the surfactant or the LDPE film during RFGD treatment.

The mechanisms responsible for the surfactant immobilization were also investigated [31]. A study of surfactants on gold substrates was designed to examine self-crosslinking in the glow-discharge-treated surfactants

An argon gas discharge treatment at $2.5 \mathrm{~W}$ and $25 \mathrm{mTorr}$ was applied to the Brij99/Au surface for $30 \mathrm{~s}$. The treated surfaces were then washed in chloroform for various soaking times. ESCA results indicated that the treated Brij99 could be removed from the Au surface only when an overnight soak in chloroform was used, while the untreated surfactant was completely removed in a $2 \mathrm{~h}$ wash. These results suggest that self-crosslinking does occur in the treated Brij99 and causes a reduction of its solubility in chloroform. However, because this self-crosslinked Brij99 can eventually be removed by soaking overnight, we conclude that self-crosslinking may not be the major mechanism for the glow discharge immobilization of the surfactant. Crosslinking between the treated (self-crosslinked) surfactant and the polymer substrate may occur and thus permanently immobilize the PEO. In addition, this study also indicates that the established washing protocol is effective in removing both the deposited Brij99 and the crosslinked surfactant.

Protein adsorption on RFGD-treated $/ \mathrm{CHCl}_{3}$-washed LDPE and Brij99/LDPE surfaces was studied using ${ }^{125}$ I-labeled baboon fibrinogen. The results are shown in Fig. 6 [26]. Fibrinogen adsorption on the RFGD-treated/ $\mathrm{CHCl}_{3}$-washed control LDPE films increased as both the treatment time and the treatment power were increased, probably as a result of the increasing surface oxidation suggested by the ESCA results. On the other hand, the RFGD-treated/ $\mathrm{CHCl}_{3}$-washed Brij99/LDPE surfaces exhibited a significant reduction in fibrinogen adsorption when a short treatment was used (less than $30 \mathrm{~s}$ ). Similar observations were also 


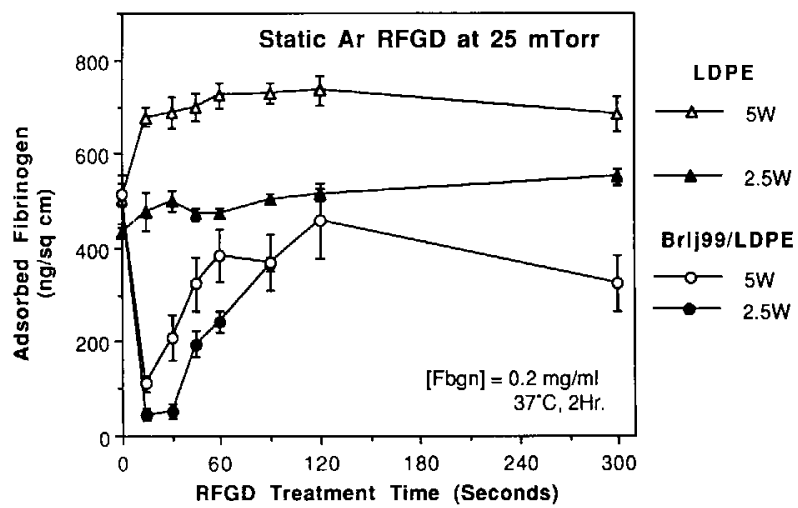

Figure 6. Fibrinogen adsorption on LDPE and Brij99/LDPE surfaces after Ar RFGD treatment and washing in chloroform [26]. Number of samples, $n$, equals 3 .

noted for the RFGD-treated/ $\mathrm{CHCl}_{3}$-washed Pluronic127/LDPE surfaces, as shown in Fig. 7 [15]. Also, as expected, the non-fouling properties of the treated surfaces are enhanced when longer PEO chains in the surfactants are used (see Fig. 7).

The results of the protein adsorption studies reveal that the PEO segments in the RFGD-immobilized surfactants exhibit non-fouling properties. Taken together with the previously described ESCA and water contact angle results, the protein adsorption studies support the proposed crosslinking of the PEO surfactants to the LDPE surface molecules via the alkyl segment of Brij99 surfactants or via the PPO segment in the Pluronic surfactants.

However, when the RFGD treatment time is prolonged, fibrinogen adsorption increases on the treated surfaces. A similar trend is also observed in platelet adhesion to the treated surfaces (see Fig. 8). SSIMS was used to investigate the

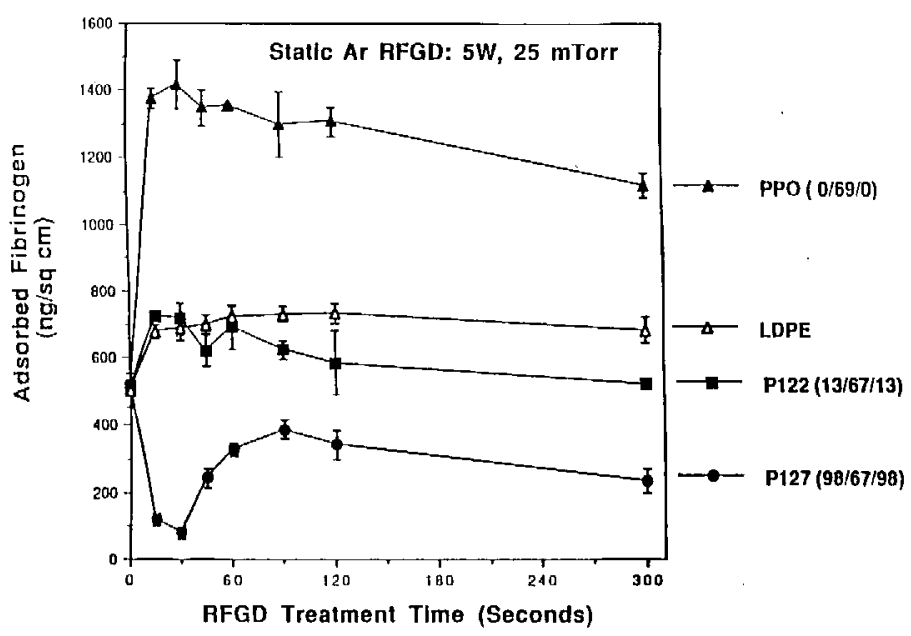

Figure 7. Fibrinogen adsorption on LDPE and Pluronic/LDPE surfaces after Ar RFGD treatment and washing in chloroform [15]. Number of samples, $n$, equals 3 . 


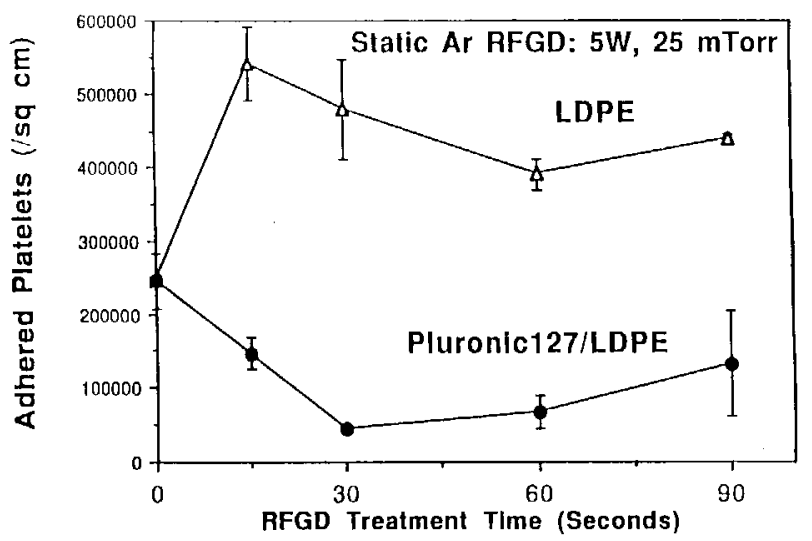

Figure 8. Platelet adhesion to LDPE and Pluronic/LDPE surfaces after Ar RFGD treatment and washing in chloroform. Number of samples, $n$, equals 3 .

possible structure changes of the PEO in the RFGD-treated surfactants [30]. Figure 9 shows the PEO index of the treated/washed surfaces as a function of the RFGD treatment time. On the treated Brij99/LDPE, a maximum in the PEO index at $30 \mathrm{~s}$ treatment is seen after $\mathrm{CHCl}_{3}$ washing. Interestingly, this curve is a mirror image of the protein adsorption and platelet adhesion curves on the treated/washed Brij99/LDPE surfaces. When the treatment time was prolonged to $120 \mathrm{~s}$, the PEO index of the treated/washed Brij99/LDPE decreased. This revealed that the relative amount of PEO chains to hydrophobic tails on the surface decreased and suggested that the PEO chains in the treated surfactant were degrading. However, when treated for $300 \mathrm{~s}$, the PEO index slightly increases again, which may have been due to the RFGD oxidation of the alkyl tails or the LDPE. The results from the SSIMS study suggest that the increases in protein adsorption and platelet adhesion at longer plasma treatment times are mainly due to the argon-plasma-induced degradation and oxidation of the PEO chains.

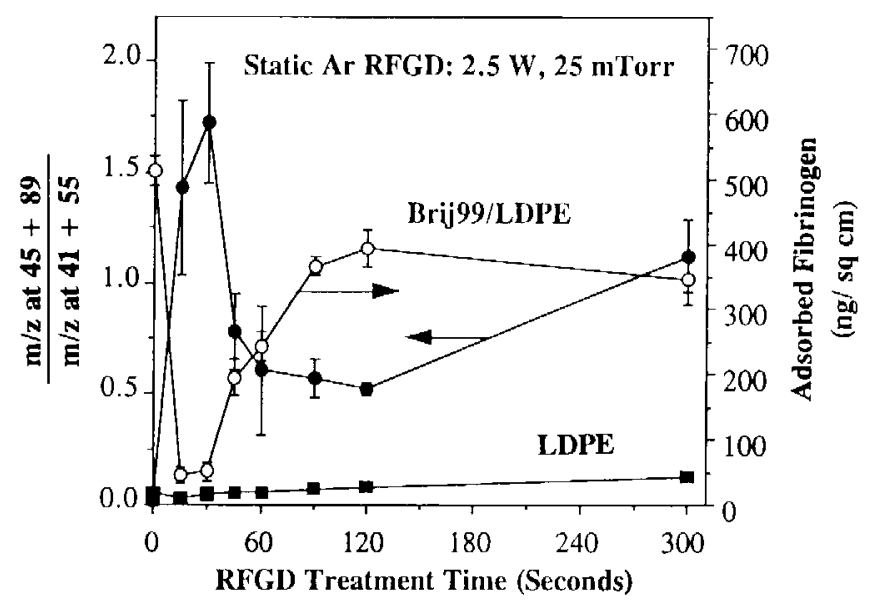

Figure 9. Static SIMS and fibrinogen adsorption for the RFGD-treated Brij99/LDPE surfaces after washing in chloroform [31]. Number of samples, $n$, equals 3 . 


\section{CONCLUSION}

Polyethylene with improved surface wettability and non-fouling (protein- and platelet-resistant) properties is obtained using a short, low power Ar RFGD treatment on the surface that has been precoated with a PEO surfactant. This glow discharge immobilization process has also been applied to functionalize polymer surfaces with sulfate groups and primary amines, when sodium dodecyl sulfate and decyl amine hydrochloride were used as the precoatings, respectively $[32,33]$. The simple glow discharge process developed in this study may have wide applicability for modifying polymer surfaces in general and biomaterial surfaces in particular.

\section{Acknowledgements}

We thank NIH (GM 40111-2, 3, and 4) and the Washington Technology Centers for their financial support for this study. We also thank NESAC/BIO at the University of Washington (NIH, RR 01296) for the use of the ESCA and static SIMS facilities, and the Regional Primate Center at the University of Washington (NIH, RR 00166) for providing fresh baboon blood for the fibrinogen adsorption and platelet adhesion experiments.

\section{REFERENCES}

1. J. D. Andrade, S. Nagaoka. S. Cooper, T. Okano and S. W. Kim, Trans. ASAIO 33, 75-84 (1987).

2. D. E. Gregonis, D. E. Buerger, R. A. Van Wagenen, S. K. Hunter and J. D. Andrade, Trans. 2nd World Congr. on Biomater. Washington, DC, pp. 266-267 (1984).

3. J. Hermans, J. Chem. Phys. 77, 2193-2203 (1982).

4. Y. Mori, S. Nagaoka, H. Takiuchi, T. Kikuchi, N. Noguchi, H. Tanzawa and Y. Noishiki, Trans. ASAIO 28, 459-463 (1982).

5. D. H. Randerson and J. A. Taylor, Plasmapheresis, New Trends in Therapeutic Applications. ISAO Press, Cleveland, OH (1983).

6. J. E. O'Mullane, C. J. Davison, K. Petrak and E. Tomlinson, Biomaterials 9, 203-204 (1988).

7. J. H. Lee, J. Kopecek and J. D. Andrade, J. Biomed. Mater. Res. 23, 351-368 (1989).

8. M. J. Bridgett, M. C. Davies and S. P. Denyer, Biomaterials 10, 411-416 (1989).

9. N. P. Desai and J. A. Hubbell, Biomaterials 12, 144-153 (1991).

10. N. P. Desai, S. F. A. Hossainy and J. A. Hubbell, Biomaterials 13, 417-420 (1992).

11. W. R. Gombotz, G. H. Wang and A. S. Hoffman, J. Appl. Polym. Sci. 37, 91-107 (1989).

12. S. W. Kim, H. Jacobs, J. Y. Lin. C. Nojori and T. Okano, Ann. N.Y. Acad. Sci. 516, 116-130 (1988).

13. Y. H. Sun, W. R. Gombotz and A. S. Hoffman, J. Bioact. Compat. Polym. 1, 316-334 (1986).

14. G. P. Lopez, B. D. Ratner, C. D. Tidwell, C. L. Haycox, R. J. Rapoza and T. A. Horbett, J. Biomed. Mater. Res. 26, 415-439 (1992).

15. M.-S. Sheu, A. S. Hoffman and J. Feijen, J. Adhesion Sci. Technol. 6, 995-1009 (1992).

16. A. Z. Okkema, T. G. Grasel, R. J. Zdrahala, D. D. Solomon and S. L. Cooper, J. Biomater. Sci. Polym. Ed. 1, 43-62 (1989).

17. D. W. Grainger, S. W. Kim and J. Feijen, J. Biomed. Mater. Res. 22, 231-249 (1988).

18. S. G. Wang, C. F. Chen, Z. F. Li, X. F. Li and H. Q. Gu, J. Macromol. Sci. Chem. A26, 505-518 (1989).

19. D. W. Grainger, C. Nojiri, T. Okano and S. W. Kim, J. Biomed. Mater. Res. 23, 979-1005 (1989).

20. K. A. Dennison, Ph.D. dissertation, Massachusetts Institute of Technology, Cambridge, MA (1986).

21. F. Epaillard, J. C. Brosse and G. Legeay, J. Appl. Polym. Sci. 38, 887-898 (1990). 
22. C. I. Simionescu. F. Denes, M. M. Macoveanu and I. Negulescu, Makromol. (hem. (Suppl.) 8, 17-36 (1984).

23. J. C. Brosse. F. Epaillard and G. Legeay, Eur. Polym. J. 19, 743-747 (1983).

24. L. H. Sharpe and H. Schonhorn, Adv. Chem. Ser. 43, 189-201 (1964).

25. R. H. Hansen and H. Schonhorn, J. Polym. Sci. B, Polym. Lett. Ed. 4, 203-209 (1966).

26. M.-S. Sheu. A. S. Hoffman, J. G. A. Terlingen and J. Feijen, Clin. Mater. (in press).

27. R. J. Rapoza and T. A. Horbett, J. Biomed. Mater. Res. 24, 1263-1287 (1990).

28. T. A. Horbett. J. Biomed. Mater. Res. 15,673-695 (1981).

29. D. X. Kiaei, A. S. Hoffman and S. R. Hanson, J. Biomed. Mater. Res. 26, 357-372 (1992).

30. M.-S. Sheu, A. S. Hoffman, J. Feijen and J. M. Harries, in preparation.

31. M.-S. Sheu, A. S. Hoffman, B. D. Ratner and J. Feijen, in preparation.

32. J. G. A. Terlingen, J. Feijen and A. S. Hoffman, J. Colloid Interface Sci. 155, 55-65 (1993).

33. J. G. A. Terlingen, L. M. Brenneisen, H. T. J. Super, A. P. Pijpers, A. S. Hoffman and J. Feijen, J. Biomater. Sci. (submitted). 